\title{
PREFACE
}

\section{Uncovering strategies to benefit from our gut microbiota: probiotics and prebiotics}

This issue of the British Journal of Nutrition publishes the proceedings of the workshop entitled 'Uncovering strategies to benefit from our gut microbiota: probiotics and prebiotics' organised by the Iberoamerican Nutrition Foundation (FINUT) and held in Valencia, Spain, on 18 April 2012, supported by the Hero Group.

Probiotics have been defined as 'live microorganisms which when administered in adequate amounts confer a health benefit on the host ${ }^{(1)}$. Lactic acid bacteria are at the historic core of probiotic health supplements and therapeutics. In particular, strains belonging to Bifidobacterium and Lactobacillus, which are the predominant groups of the gastrointestinal microbiota, are the most widely used probiotic bacteria and are included in many functional foods and dietary supplements; other micro-organisms such as moulds and yeasts, namely Saccharomyces boulardii yeast, has also been shown to have health benefits ${ }^{(2,3)}$.

The use of fermented foods for prevention and treatment of diseases is very ancient, and noted health qualities of yogurt, kefir, koumis and other fermented milk products were well known to the peoples of the Middle East and Asia and predate more modern observations by perhaps 5000 years. The more modern concepts of probiotics have their inception in the works of the Nobel Prize winner Ilya Mechnikov (1845-1916) who argued in favour of the health benefits of Bulgarian yogurt and identified its active micro-organisms and promoted the idea that yogurt and its bacterial constituents were essential ingredients contributing to the longevity seen in Bulgarian peasants ${ }^{(4)}$. After a long history of safe use of probiotics in fermented dairy products and an increased recognition of their beneficial effects on human health, the food industry has become increasingly interested in these types of micro-organisms.

The results of evidence-based analyses from human studies and animal models have shown the potential clinical effectiveness of probiotics against many diseases ${ }^{(1-3)}$. Probiotics have been reported to suppress diarrhoea, alleviate lactose intolerance and post-operative complications, exhibit antimicrobial activities and anti-colorectal cancer activities, reduce irritable bowel symptoms and prevent inflammatory bowel disease. However, generalisations concerning the potential health benefits of probiotics should not be made because probiotic effects tend to be strain-specific. Moreover, it is important to note that many probiotic health claims have not yet been substantiated by experimental evidence. In addition, the efficacy demonstrated for one given bacterial strain cannot necessarily be transferred to other probiotic organisms. Moreover, the mechanisms underlying probiotic action have not yet been fully elucidated ${ }^{(5)}$.

Several important mechanisms underlying the antagonistic effects of probiotics on various micro-organisms include the following mechanisms: modification of the gut microbiota; competitive adherence to the mucosa and epithelium; strengthening of the gut epithelial barrier; modulation of the immune system to convey an advantage to the host ${ }^{(5)}$. The recent characterisation of the host families of pattern recognition molecules, such as Toll-like receptor and nucleotide oligomerisation domain-like receptors, as well as modulating key signalling pathways, such as NF- $\mathrm{BB}$ and mitogen-activated protein kinase, with respect to their ability to enhance or suppress activation and influence downstream pathways will shed light onto the complex interplay of host-microbe interactions and, particularly, on the gut-associated lymphoid tissue and immune responses. Stimulation of these receptors by commensal bacteria has a crucial role to elicit measured antimicrobial responses with minimal inflammatory tissue damage ${ }^{(5,6)}$.

In the present supplement, the mechanisms involving host-microbe interactions and gut immune system and oral tolerance and how gut microbiota is related to human health are reviewed. Moreover, sources, isolation, characterisation and evaluation of probiotics are described. Particularly, we pay attention to the isolation, identification characterisation, safety and competitive inhibition against enteropathogens of three novel probiotic strains (Lactobacillus paracasei CNCM I-4034, Bifidobacterium breve CNCM I-4035 and Lactobacillus rhamnosus CNCM I-4036) from the faeces of exclusively breast-fed infants. In addition, the scientific evidence from a paediatric perspective of the clinical effects of probiotics is summarised. Likewise, we describe major relationships among host genotype, intestinal microbiota and inflammatory disorders.

Prebiotic is a concept that refers to non-digestible food ingredients with selective effects on the intestinal microbiota. Oligosaccharides are the main components, and the diverse range of materials may be based on any of the hexose monosaccharides, including glucose, fructose, galactose and mannose with a polymerisation degree between 2 and 20 monosaccharides $^{(4)}$. In the present supplement of the British Journal of Nutrition, we also describe how changes in the 
gut microbiota composition and activity by dietary fibres with prebiotic properties can modulate host gene expression and metabolism and how prebiotic can work in the management of obesity and related metabolic disturbances, in view of the experimental data and intervention studies published up to date.

The molecular elucidation of probiotic and prebiotic actions in vivo will help to identify true probiotics and prebiotics and to select the most suitable ones for the prevention and treatment of particular diseases.

Angel Gil

Department of Biochemistry and Molecular Biology II, Institute of Nutrition and Food Technology "Jose Mataix", Biomedical Research Centre, University of Granada, Avenida Conocimiento s/n 18100 Armilla,

Granada, Spain

doi:10.1017/S0007114513000081

\section{References}

1. Reid G, Sanders ME, Gaskins HR, et al. (2003) New scientific paradigms for probiotics and prebiotics. J Clin Gastroenterol 37, 105-118.

2. Liong MT (2011) Probiotics: Biology, Genetics and Health Aspects. Microbiology Monographs. Heidelberg: Springer.

3. Floch MH, Walker WA, Madsen K, et al. (2011) Recommendations for probiotic use-2011 update. J Clin Gastroenterol 45, S168-S171.

4. Hume ME (2011) Historic perspective: prebiotics, probiotics, and other alternatives to antibiotics. Poult Sci 90, $2663-2669$

5. Bermudez-Brito M, Plaza-Díaz J, Muñoz-Quezada S, et al. (2012) Probiotic mechanisms of action. Ann Nutr Metab 61, $160-174$

6. Bermudez-Brito M, Muñoz-Quezada S, Gomez-Llorente C, et al. (2012) Human intestinal dendritic cells decrease cytokine release against Salmonella infection in the presence of Lactobacillus paracasei upon TLR activation. PLOS One 7 , e43197. 